\title{
Structures of MHC-I/Tapasin and MHC-I/TAPBPR Describe the Mechanism of Peptide Loading Antigen Presentation
}

\author{
J Jiang ${ }^{1}$, K Natarajan ${ }^{2}$, E Kim ${ }^{2}$, J Dhobi ${ }^{2}$, N Sherani ${ }^{2}$, M Mage $^{2}$, L Boyd ${ }^{2}$, D Margulies ${ }^{2}$ \\ ${ }^{1}$ NIAID/NIH, Bethesda, MD, ${ }^{2}$ LISB/NIAID/NIH, Bethesda, MD \\ jiangji@niaid.nih.gov
}

MHC molecules of the class I type (MHC-I) bind to endogenous peptides in the endoplasmic reticulum and the peptide-loaded MHC-I molecules are subsequently transported to the cell surface where they serve as indispensable ligands for T cell and NK cell development and function. Peptide loading occurs in the ER within the peptide loading complex (PLC) in which critical aspects of MHC-I stabilization and peptide loading are dependent on the chaperone, tapasin. However, MHC-I molecules can also be loaded independently of the PLC by a tapasin homolog designated TAPBPR. To elucidate mechanistic aspects of PLC-dependent and independent peptide loading we have determined the X-ray crystal structures of MHC-I with tapasin and compare that with previously reported the structure of a MHC-I/TAPBPR complex (Jiang, et al., Science 2017, 358, 1064-68). These structures capture distinct chaperone-stabilized MHC-I conformation, and provide insight into the mechanism of PLC-dependent and PLC-independent MHC-I peptide loading. (Supported by the Intramural research program of the NIAID, NIH)

Acta Cryst. (2020). A76, a39 